\title{
EFFECT OF ACID NEUTRALIZER TOOTHPASTE ON SALIVARY pH IN A GROUP OF EGYPTIAN CHILDREN: A RANDOMIZED CONTROLLED PILOT STUDY
}

\author{
Fatma Abdelgawad*, Nada Wassef* and Ahmed Salama*
}

\begin{abstract}
Aim or purpose: Dental caries is a preventable disease that affects the quality of life and overall health. Cariogenic carbohydrates are the main cause. Proper toothbrushing with toothpaste helps in caries prevention. The aim of this study is to compare the effect of acid neutralizer toothpaste and regular toothpaste on salivary $\mathrm{pH}$ in a group of Egyptian children.

Materials and methods: Twenty children with mixed dentition were randomly allocated either to the control or test group. Saliva samples were collected from each child for baseline $\mathrm{pH}$ measurement using $\mathrm{pH}$ meter. Children were instructed to drink $200 \mathrm{ml}$ of juice and saliva samples were collected. They brushed their teeth with either Colgate maximum cavity protection with sugar acid neutralizer toothpaste ${ }^{\circledR}$ (test group) or the Colgate total toothpaste ${ }^{\circledR}$ (control group) then saliva samples were collected again. The results were tabulated and statistically analyzed.
\end{abstract}

Results: For both groups, there was a drop in $\mathrm{pH}$ levels after drinking juice. The mean $\mathrm{pH}$ value dropped from $6.90 \pm 0.29$ to $6.78 \pm 0.43$ for the test group and from $7.01 \pm 0.38$ to $6.88 \pm 0.59$ for the control group. After brushing, the mean $\mathrm{pH}$ values elevated for both groups. The mean $\mathrm{pH}$ for the test and control groups was $7.24 \pm 0.28$ and $7.26 \pm 0.30$ respectively after brushing. There was a statistically significant difference between the $\mathrm{pH}$ levels within each group before and after brushing. However, there was no statistically significant difference between both groups.

Conclusion: Toothbrushing after drinking juice results in elevation of the $\mathrm{pH}$ level which may be helpful in reducing dental caries.

KEYWORDS: Toothbrushing, toothpaste, acid neutralizer, salivary $\mathrm{pH}$, dental caries

\section{INTRODUCTION}

Dental caries is considered a major public health problem. In 2014, the Ministry of Health and population and the WHO country office surveyed the oral health status of over 10,000 citizens in the 27 Egyptian governorates. The survey results stated that almost $70 \%$ of the surveyed children had untreated caries (WHO EMRO, 2014).

\footnotetext{
* Lecturer, Pediatric Dentistry and Dental Public Health, Faculty of Dentistry, Cairo University.
} 
Among children, dental caries can have serious results affecting the child's general health and quality of life. According to Mohammadi \& Kay, 2012 , children's growth is affected by the state of their dental health. Gilchrist et al., 2015 reported the effect of caries on children that may affect their quality of life including pain, difficulty in eating, sleeping disturbances, emotional impacts, and effects as a result of poor esthetics.

Dental caries is the result of interaction between cariogenic bacteria, mainly Streptococcus Mutans and Lactobacilli, with freely fermentable carbohydrates resulting in demineralization of the inorganic components of the tooth and subsequent destruction of the organic part leading to cavitation (Arends, 1982). Therefore, acid production is considered a detrimental part in the carious process. Toothbrushing and the use of toothpaste is an important preventive measure in the caries process as it results in the disruption of the bacterial biofilm (Topping \& Assaf, 2005).

A toothpaste recently introduced by Colgate, namely Colgate Maximum cavity protection with Sugar Acid Neutralizer Toothpaste ${ }^{\circledR}$, consists of arginine in insoluble calcium base causing neutralization of sugar acids in plaque and saliva. Thus, decreasing dental caries.

Among children, the consumption of sugar-containing juices is frequent and can result in increased caries risk with its unfavorable consequences on the child's health. So, the aim of this study is to compare the effect of the new Colgate maximum cavity protection with sugar acid neutralizer toothpaste ${ }^{\circledR}$ with the conventional toothpaste after consumption of juice, to evaluate its effect on acids.

\section{PARTICIPANTS AND METHODS}

\section{Research ethics committee approval}

Ethical approval was obtained from the research ethics committee, Faculty of Dentistry, Cairo University. Twenty children were selected from the outpatient clinic, Pediatric Dentistry and Dental Public Health Department, Faculty of Dentistry, Cairo University. A written informed consent was obtained from the parents after explanation of the procedures and verbal assent was obtained from their children.

\section{Study sample:}

Sample size: this study is a pilot study because of the lack of studies available in the literature for sample size calculation. There was only one study by $\mathrm{Cg}$ et al., 2016 with a different study group (adults). The sample size was estimated to be 20 children (10 per group) according to Hertzog, 2008.

\section{Sample type:}

A convenience consecutive sampling was used. Children satisfying the eligibility criteria attending the outpatient clinic, Pediatric Dentistry and Dental Public Health Department, Faculty of Dentistry, Cairo University were included in the study.

\section{Eligibility criteria:}

Children with mixed dentition, both genders were included, patients with no medical diseases or on medications and patients with DMF $>1$.

The children were randomly allocated either to the test or control group.

\section{Randomization:}

Sequence generation was done using online generator website (random.org), where two groups were randomly generated each with ten children. The generated sequences were then allocated to the test and control groups. The generated sequences were written in a paper and folded four times and kept in sealed opaque envelopes in a bowl. The sequences generated were performed and kept with one of the researchers who is not involved in the trial conduction. Then, the child chooses an envelope from the bowl and was assigned either to the test or control group. 


\section{Blinding}

This is a double-blind study, were the children or their parents and assessor were blinded to the toothpaste type and whether the saliva sample was for a test or control group. One researcher was blinded to provide the juice as well as the toothpaste without knowing if the toothpaste is from the test or control group and collected the saliva samples. Another researcher measured the salivary $\mathrm{pH}$. For each child, a diagnostic chart was filled with full medical and dental histories, and the teeth were examined.

\section{The procedure}

For each child, baseline saliva sample was collected in a cup by asking the child to spit. The baseline salivary $\mathrm{pH}$ was measured using digital $\mathrm{pH}$ meter. Children were instructed to drink $200 \mathrm{ml}$ of apple juice and then saliva samples were collected again from each child for $\mathrm{pH}$ measurement. The $\mathrm{pH}$ meter was neutralized after each measurement using distilled water to avoid contamination of the measured sample.

Each child was provided with a toothbrush and a pea-sized amount of toothpaste either the test or control group. Children were instructed to brush their teeth with the toothpastes and then saliva samples were collected for $\mathrm{pH}$ measurement. Children were guided to use the Fone's toothbrushing method as it is the easiest method for kids. The results were then tabulated and statistically analyzed using IBM SPSS version 22. One-way ANOVA was used for testing of significance within groups and between groups. Post hoc was done using LSD.

\section{RESULTS}

Twenty children were included in the study, with a mean age of $8.55 \pm 1.48$ years for group (1) (Colgate sugar acid neutralizer toothpaste group) and mean age of 9.4 \pm 2.32 years for group (2) (Colgate total toothpaste group). The mean DMF for group (1) and group (2) was 1.3 and 1.7, respectively. The mean def for group (1) and group (2) was 5.2 and 4.4, respectively.

The mean baseline $\mathrm{pH}$ of both groups was $6.90 \pm 0.29$ and $7.01 \pm 0.38$ for group (1) and (2) respectively. After drinking the fruit juice, the mean $\mathrm{pH}$ of both groups was $6.78 \pm 0.43$ and $6.88 \pm 0.59$ for group (1) and (2) respectively. After toothbrushing, the mean $\mathrm{pH}$ for both groups was $7.24 \pm 0.28$ and $7.26 \pm 0.30$ respectively as shown in figure (1).

One-way ANOVA revealed that there is a statistically significant difference with a $P$ value 0.04 . Post hoc analysis was done to assess the significance. Regarding the mean baseline $\mathrm{pH}$ for both groups, there was no statistically significant difference between the two groups with a $P$ value 0.51 . In both groups, there was a drop in $\mathrm{pH}$ values after drinking the fruit juice. However, the $\mathrm{pH}$ level was elevated again after toothbrushing in both groups.

Within each group, there was a statistically significant difference between the mean $\mathrm{pH}$ after drinking the juice and after brushing with a $P$ value 0.01 and 0.04 for group (1) and (2) respectively.

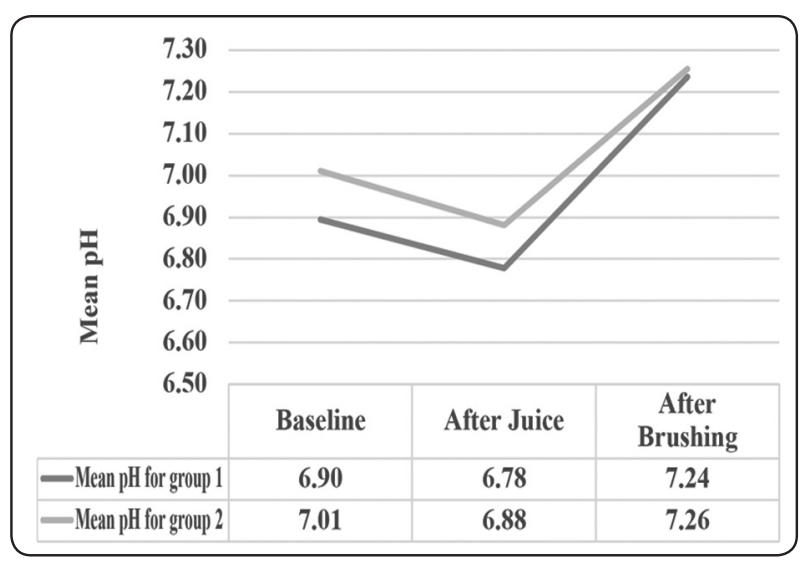

Fig. (1): Mean $\mathrm{pH}$ values for both groups. 


\section{DISCUSSION}

In this study, the aim is to evaluate the effect of the Colgate maximum cavity protection with sugar acid neutralizer toothpaste ${ }^{\circledR}$ in elevation of the acidic $\mathrm{pH}$ resulting from drinking juices as this is common in children.

According to Marsh, 2006, cariogenic bacteria are present in low numbers in plaque without causing disease in the normal conditions. Ecological changes of the dental plaque (as sugar-rich diet, conditions that lower the $\mathrm{pH}$, or reduced salivary flow) can result in dominance of these cariogenic species and dental caries results. Therefore, prevention of the caries process can be achieved not only by inhibition of the micro-organisms directly (e.g. with antimicrobial or anti-adhesion agents) but also indirectly by alteration of the ecological conditions responsible for enhancing the caries process.

The selection of the Colgate maximum cavity protection with sugar acid neutralizer toothpaste ${ }^{\circledR}$ aimed to determine whether it is effective in raising the $\mathrm{pH}$ which can be protective against enamel demineralization. Fluoride has been the main protective component in dentifrices; however, fluoride has limitations as it does not target dental plaque as the main mode of action (Cg et al., 2016).

The introduction of Colgate sugar acid neutralizer toothpaste ${ }^{\circledR}$ can also be helpful in cases where patients suffer from gastric regurgitation as it is acidic and can be devastating to the tooth structure and can result in erosion as stated by Dawes, 2003.

The toothpaste characteristics that greatly affect their cosmetic or therapeutic effect are their physical form, chemical composition, their $\mathrm{pH}$, and their solubility according to Kalyana, 2014. Also, the $\mathrm{pH}$ of the saliva is a key factor in the carious process as it affects the demineralization and remineralization processes of teeth (Bartlett et al., 2003). When the $\mathrm{pH}$ in the oral cavity decreases, hydroxyapatite dissolution or demineralization occurs and when
$\mathrm{pH}$ is elevated, the hydroxyapatite precipitation or remineralization occurs (Mary et al., 2018). Therefore, the idea of sugar acid neutralization can be helpful in arresting the caries process.

The study is a pilot study due to the lack of literature addressing this topic, making it difficult to calculate the sample size. The toothpaste used as control is the Colgate total toothpaste as it is available and from the same manufacturer.

Blinding was readily achieved in this study as children were not able to differentiate the toothpastes types as they have similar color and appearance. The assessor was also blinded as he wasn't the same researcher giving the patients the toothpaste and does not know if the saliva sample belongs to the test or control group.

The age of children helped to control proper toothbrushing and spitting. The mean DMF and def scores of the two groups were not statistically significant, this was useful to standardize between both groups.

The results showed that the mean baseline $\mathrm{pH}$ values were not statistically significant between both groups, which is also considered as an indication of standardization between both groups.

Upon drinking the fruit juice, the $\mathrm{pH}$ dropped significantly and was elevated again after brushing with either of the toothpastes which is in accordance with the results of Soham et al., 2015 and Mary et al., 2018. This indicates the importance of brushing in raising the $\mathrm{pH}$ after intake of sugar.

However, there was no statistically significant difference between the mean $\mathrm{pH}$ values of the two groups. Further studies are needed to compare the mean $\mathrm{pH}$ values upon using the Colgate maximum cavity protection with sugar acid neutralizer toothpaste and other types of toothpastes with a larger sample size. 


\section{CONCLUSION}

Toothbrushing after drinking juice results in elevation of the $\mathrm{pH}$ level which may be helpful in reducing dental caries.

\section{REFERENCES}

1. Arends, J. (1982) Mechanism of Dental Caries. Biological Mineralization and Demineralization, pp. 303-324.

2. Bartlett, D. W., Bureau, G. P. \& Anggiansah, A. (2003) Evaluation of the PH of a New Carbonated Soft Drink Beverage: An in Vivo Investigation. Journal of Prosthodontics: Implant, Esthetic, and Reconstructive Dentistry, 12 (1), pp. 21-25.

3. Cg, A. K., Gurnani, P., Shah, A., Ghosh, A., Shrivastava, H. \& Gurnani, R. (2016) Effect of Commercially Available Colgate Sugar Acid Neutralizer Toothpaste on Salivary and Plaque Ph: A Randomized Controlled Trial.

4. Dawes, C. (2003) What Is the Critical PH and Why Does a Tooth Dissolve in Acid? Journal (Canadian Dental Association), 69 (11), pp. 722-724.

5. Gilchrist F, Marshman Z, Deery C, Rodd H. (2015) The impact of dental caries on children and young people: What they have to say?. Int J Paediatr Dent., 25(5); p.327-38.
6. Hertzog, M. A. (2008) Considerations in Determining Sample Size for Pilot Studies. Research in Nursing \& Health, 31 (2), pp. 180-191.

7. Kalyana, C. (2014) Dental indices: ready reckoner (hb). Cbs publishers \& distribu.

8. Marsh, P. D. (2006) Dental Plaque as a Biofilm and a Microbial Community - Implications for Health and Disease. BMC oral health, 6 Suppl 1 (Suppl 1), p. S14.

9. Mary, D., Vishnu Priya, V. \& Gayathri, R. (2018) Effects of Toothpaste and Mouthwash on Salivary PH in Adolescents. Drug Invention Today, 10 (9), pp. 1731-1733.

10. Mohammadi, T. M. \& Kay, E. J. (2012) Effect of Dental Caries on Children Growth. In: Li, D. M.-Y. ed., Contemporary Approach to Dental Caries. InTech, pp. 379-394.

11. Soham, B., Srilatha, K. \& Seema, D. (2015) Effects of Fluoridated Toothpaste and Mouth Rinse on Salivary PH in Children- An In Vivo Study. Journal of Oral Hygiene \& Health, 03 (06) November, pp. 1-7.

12. Topping, G. \& Assaf, A. (2005) Strong Evidence That Daily Use of Fluoride Toothpaste Prevents Caries How Effective Is Fluoride Toothpaste in Preventing Caries? Evidence-Based Dentistry, 6, p. 32.

13. WHO EMRO (2014) Egypt Releases Results of Epidemiological Study on Oral Health Status. 